\title{
Early MoCA predicts long-term cognitive and functional outcome and mortality after stroke
}

Vera Zietemann, PhD, * Marios K. Georgakis, MD, MSc,* Thibaut Dondaine, PhD, Claudia Müller, MD, Anne-Marie Mendyk, RN, Anna Kopczak, MD, Hilde Hénon, MD, PhD, Stéphanie Bombois, MD, PhD, Frank Arne Wollenweber, MD, Régis Bordet, MD, PhD, and Martin Dichgans, MD

Neurology ${ }^{\circledR}$ 2018;91:e1838-e1850. doi:10.1212/WNL.0000000000006506

\section{Abstract}

\section{Objective}

To examine whether the Montreal Cognitive Assessment (MoCA) administered within 7 days after stroke predicts long-term cognitive impairment, functional impairment, and mortality.

\section{Methods}

MoCA was administered to 274 patients from 2 prospective hospital-based cohort studies in Germany $(\mathrm{n}=125)$ and France $(\mathrm{n}=149)$. Cognitive and functional outcomes were assessed at 6,12 , and 36 months after stroke by comprehensive neuropsychological testing, the Clinical Dementia Rating (CDR) scale, the modified Rankin Scale (mRS), and Instrumental Activities of Daily Living (IADL) and analyzed with generalized estimating equations. All-cause mortality was investigated by Cox proportional hazard models. Analyses were adjusted for demographic variables, education, vascular risk factors, premorbid cognitive status, and NIH Stroke Scale scores. The additive predictive value of MoCA was examined with receiver operating characteristic curves.

\section{Results}

In pooled analyses, a baseline MoCA score <26 was associated with cognitive impairment, defined by neuropsychological testing (odds ratio [OR] 5.30, 95\% confidence interval [CI] 2.75-10.22) and by CDR score $\geq 0.5$ (OR 2.53, 95\% CI 1.53-4.18); functional impairment, defined by $\mathrm{mRS}$ score $>2$ (OR 5.03, 95\% CI 2.20-11.51) and by IADL score <8 (OR 2.48, 95\% CI 1.40-4.38); and mortality (hazard ratio 7.24, 95\% CI 1.99-26.35) across the 3-year followup. Patients with MoCA score $<26$ performed worse across all prespecified cognitive domains (executive function/attention, memory, language, visuospatial ability). MoCA increased the area under the curve for predicting cognitive impairment (neuropsychological testing 0.81 vs $0.72, p=0.01$ ) and functional impairment ( $\mathrm{mRS}$ score $>2,0.88$ vs $0.84, p=0.047)$.

\section{Conclusion}

Early cognitive testing by MoCA predicts long-term cognitive outcome, functional outcome, and mortality after stroke. Our results support routine use of the MoCA in stroke patients.

\author{
Correspondence \\ Dr. Dichgans \\ martin.dichgans@ \\ med.uni-muenchen.de
}

\section{RELATED ARTICLE}

\section{Editorial}

Independence after stroke:

Mind over matter

Page 903

*These authors contributed equally to this work.

From the Institute for Stroke and Dementia Research (V.Z., M.K.G., C.M., A.K., F.A.W., M.D.), University Hospital, Ludwig-Maximilians-University, Munich, Germany; University of Lille (T.D., A.-M.M., H.H., S.B., R.B.), Inserm, CHU Lille, "Degenerative and Vascular Cognitive Disorders", Lille, France; German Centre for Neurodegenerative Diseases (M.D.); and Munich Cluster for Systems Neurology (M.D.), Germany.

Go to Neurology.org/N for full disclosures. Funding information and disclosures deemed relevant by the authors, if any, are provided at the end of the article. 


\section{Glossary}

AUC $=$ area under the ROC curve $\mathbf{C D R}=$ Clinical Dementia Rating; $\mathbf{C I}=$ confidence interval DEDEMAS $=$ Determinants of Dementia After Stroke; IADL = Instrumental Activities of Daily Living; IQCODE = Informant Questionnaire on Cognitive Decline in the Elderly; MoCA = Montreal Cognitive Assessment; mRS = modified Rankin Scale; NIHSS = NIH Stroke Scale; OR = odds ratio; ROC = receiver operating characteristic; STROKDEM = Study of Factors Influencing Post-Stroke Dementia; TOAST $=$ Trial of Org 10172 in Acute Stroke Treatment.

The growing number of stroke survivors ${ }^{1,2}$ has sparked interest in the long-term consequences of stroke and the prediction of outcomes. Cognitive impairment is found in up to $90 \%$ of stroke survivors depending on time interval after the event, ${ }^{3,4}$ affects quality of life, ${ }^{5}$ and predicts dependency ${ }^{6}$ and death. ${ }^{7}$ Current expert statements and guidelines recommend formal cognitive testing after stroke. ${ }^{8}$ However, little is known about the predictive value of early cognitive testing for longterm outcome after stroke.

The Montreal Cognitive Assessment (MoCA) has gained wide acceptance for use in stroke patients because of its brevity, ${ }^{8,9}$ sensitivity to mild cognitive impairment, ${ }^{10,11}$ feasibility, and retained validity in the acute and subacute setting. ${ }^{12,13}$ Previous studies have investigated the utility of the MoCA during hospitalization to predict cognitive ${ }^{14-17}$ or functional outcome ${ }^{18}$ up to 12 months after stroke. However, these studies included patients with TIA, ${ }^{14,17,18}$ did not require imaging confirmation of stroke, ${ }^{14,16-18}$ or had small sample size ${ }^{15}$ or short followup. ${ }^{15,16,18}$ In addition, it is unknown whether baseline cognitive performance predicts mortality after stroke.

We thus set out to investigate whether the MoCA administered within 7 days after stroke predicts long-term outcome independently from premorbid cognitive status, demographic characteristics, and stroke severity. Specifically, we determined the predictive value of baseline MoCA for cognitive and functional impairment and for all-cause mortality. We further explored whether baseline MoCA predicts performance in individual cognitive domains. To address these aims, we leveraged data from 2 prospective hospital-based cohort studies with harmonized study protocols and serial assessments up to 3 years after stroke.

\section{Methods}

\section{Study populations and study design}

Participants were drawn from 2 prospective hospital-based cohort studies; the Determinants of Dementia After Stroke (DEDEMAS) study (NCT01334749) conducted at a tertiary care university hospital at Ludwig-Maximilians-Universität, Munich, Germany, and the Study of Factors Influencing PostStroke Dementia (STROKDEM) study (NCT01330160) conducted at the Lille University Hospital, France. DEDEMAS and STROKDEM had been planned in parallel with harmonization of study protocols regarding inclusion and exclusion criteria, data collection, and follow-up. Details of the study designs have previously been described. ${ }^{19,20}$ In brief, we recruited adult patients presenting with an acute stroke defined by a focal neurologic deficit in combination with a corresponding infarct on brain MRI. We excluded patients with a diagnosis of dementia or a summed score of $>64$ in the short version of the Informant Questionnaire on Cognitive Decline in the Elderly (IQCODE), ${ }^{21}$ known diseases of the CNS other than stroke, a condition interfering with follow-up such as end-stage malignancy, or missing language skills.

Patients were enrolled between May 2011 and November 2013 in DEDEMAS and between January 2010 and April 2014 in STROKDEM. The participation rates for the 2 cohorts, as calculated from the total number of patients with a final diagnosis of stroke examined in the respective hospitals during the enrollment period, were 6\% for DEDEMAS and 5\% for STROKDEM. Baseline assessments included demographic data, a complete medical history, details on hospitalization, and a complete neurologic examination, including the $\mathrm{NIH}$ Stroke Scale (NIHSS) ${ }^{22}$ Stroke was classified as ischemic or hemorrhagic, and ischemic strokes were further subclassified according to the modified Trial of Org 10172 in Acute Stroke Treatment (TOAST) criteria. $^{23}$ In DEDEMAS, we further applied the Delirium Rating Scale-Revised-98, and a score $\geq 16$ was used to define delirium.

\section{MoCA at baseline}

Baseline cognitive status was assessed by the MoCA, a screening tool for global cognition ${ }^{9}$ that has been shown to retain its validity after stroke. ${ }^{12,13,24}$ The MoCA was administered during hospitalization within 7 days after stroke symptom onset. Cognitive impairment was defined by a MoCA score <26, a cutoff previously validated in stroke patients. ${ }^{10,24,25}$ To control for educational status, we added 1 point to the MoCA score in patients with $<12$ years of education. ${ }^{9}$ Patients with missing items on the MoCA at baseline were included in the study only if their score remained $<26$ when giving the maximum possible score for missing items and were thus definitely classified as cognitively impaired or if they had already scored $\geq 26$ in the completed items and were thus definitely classified as not cognitively impaired. Patients without a MoCA assessment within 7 days after stroke at all or patients with missing items in MoCA not fulfilling the above criteria were excluded from the current study.

\section{Follow-up and assessment of outcomes}

All patients underwent a comprehensive evaluation of cognitive and functional outcome by face-to-face interviews with 
the patient and the informant during follow-up visits at 6,12 , and 36 months after stroke.

\section{Cognitive outcome}

An extensive battery of neuropsychological tests, broadly classified in 4 cognitive domains (executive function/ attention, memory, language, visuospatial ability; data available from dryad, table e-1, doi.org/10.5061/dryad.7h083rd) were administered to patients at each follow-up time point. For every patient, we calculated test-specific $z$ scores on the basis of published norms corrected for age, sex, and education. We further obtained summary domain-specific $z$ scores by averaging the test-specific $z$ scores in each domain. ${ }^{26}$ A summary $z$ score $\leq 1.5$ in at least 1 domain was used to determine cognitive impairment. ${ }^{26}$ As an alternative measure of cognitive impairment, we used the Clinical Dementia Rating (CDR) scale. $^{27}$ Face-to-face interviews were conducted with the patient and an informant, and global scores were computed. We defined cognitive impairment as a global score $\geq 0.5$. ${ }^{28}$

\section{Functional outcome}

Functional outcome was assessed with the modified Rankin Scale (mRS), a global scale focusing primarily on motor function. ${ }^{29}$ Scores range from 0 (no symptoms) to 6 (death), and the interview was conducted with the patient or informant in case of poor condition of the patient. A score $>2$ defined functional impairment. ${ }^{30,31}$ The ability to carry out more complex activities was assessed with the Lawton Instrumental Activities of Daily Living (IADL) scale, ${ }^{32}$ which has been suggested as a measure of functional outcome in stroke survivors. $^{31,33}$ The interview was conducted with an informant. The scale evaluates independence in 8 daily tasks, and scores range from 0 to 8 . Loss of independence in any of the tasks (score <8) determined functional impairment. ${ }^{31}$

\section{All-cause mortality}

Information on vital status and date of death up to 3 years after stroke were obtained for all patients who did not attend the follow-up visits either through telephone interviews with the informants or general practitioners or from the registry offices.

\section{Standard protocol approvals, registrations, and patient consent}

DEDEMAS and STROKDEM were conducted according to the Declaration of Helsinki and were approved by the local ethics committees. ${ }^{19}$ Written informed consent was obtained by all patients or legal guardians before participation. Approval for surrogate consent was obtained to minimize recruitment bias.

\section{Statistical analysis}

Differences in the patient characteristics between DEDEMAS and STROKDEM were evaluated with the $\chi^{2}$ or the Fisher exact test for categorical variables. For continuous variables, we used a 2-tailed $t$ test or Mann-Whitney $U$ test, as appropriate.

We fitted generalized estimating equations models ${ }^{34}$ to calculate the association of the baseline MoCA score ( $<26$ vs $\geq 26$ ) with the cognitive and functional outcomes at 6,12 , and 36 months after stroke. Binary outcomes (cognitive impairment $[z$ score $\leq 1.5$ in $\geq 1$ domain or CDR score $\geq 0.5]$ and functional impairment $[\mathrm{mRS}$ score $>2$ or IADL score $<8]$ ) were assessed by logistic models. For continuous outcomes (summary $z$ score in executive function/attention, memory, language, and visuospatial ability), we implemented generalized linear models. Temporal progression of cognitive and functional impairment was evaluated by including time in the models. To examine outcomes at single time points, we performed logistic regression and linear regression analyses for binary and continuous outcomes, respectively. Mortality was assessed by Cox proportional hazard models. Odds ratios (ORs) and unstandardized effect estimates were derived for the binary and continuous outcomes, respectively, and hazard ratios were computed for mortality. Analyses were adjusted for age, sex, education, history of hypertension and diabetes mellitus (defined by physician diagnosis or prescription of antihypertensive or antidiabetic agents), baseline IQCODE score, and NIHSS score at admission. In sensitivity analyses, we excluded patients with a history of stroke, with subthreshold delirium symptoms (conducted in DEDEMAS for data availability), and with hemorrhagic stroke and further adjusted for APOE genotype (conducted in DEDEMAS for data availability). We further stratified our analyses by baseline stroke severity according to admission NIHSS score ( $\leq 3$ vs $>3) .{ }^{35}$ Analyses were conducted separately for DEDEMAS and STROKDEM. Pooled results were analyzed with fixedeffects meta-analyses. In the presence of heterogeneity $\left(I^{2} \geq\right.$ $50 \%$ or $p$ from Cochran $\mathrm{Q}<0.10)$, random-effects metaanalysis was used. ${ }^{36}$ To account for multiple comparisons, we used the false discovery rate, setting statistical significance level at $<0.05$.

To examine the additive predictive value of baseline MoCA on top of the known predictive ability of the NIHSS at admission, ${ }^{37,38}$ we compared the areas under the receiver operating characteristic (ROC) curve (AUC; C statistic) in (1) a basic model adjusted for age, sex, education, history of hypertension, history of diabetes mellitus, and baseline IQCODE (model 1); (2) model 1 plus NIHSS score at admission (model 2); and (3) model 2 plus baseline MoCA score ( $<26$ vs $\geq 26$, model 3 ). Optimal cutoffs for predicting cognitive or functional impairment were determined by use of the entire range of MoCA scores as a continuous variable and calculating the Youden Index. All analyses were carried out with SAS version 9.4 (SAS Institute Inc, Cary, NC).

\section{Data availability}

Data can be made available on reasonable request. The requests should be addressed to the principal investigators of DEDEMAS (M. Dichgans, martin.dichgans@med.unimuenchen.de) and STROKDEM (R. Bordet, regis.bordet@ univ-lille.fr). Because of restrictions based on privacy regulations and informed consent of the participants, data cannot be made freely available in a public repository. 
Table 1 Baseline characteristics of the 2 study cohorts

\begin{tabular}{|c|c|c|c|c|}
\hline & DEDEMAS $(n=125)$ & STROKDEM $(n=149)$ & Pooled data $(n=274)$ & $p$ Value \\
\hline \multicolumn{5}{|l|}{ Demographic variables } \\
\hline Age, y & $70.2 \pm 8.1$ & $64.4 \pm 12.8$ & $67.0 \pm 11.3$ & $<0.0001$ \\
\hline Female, n (\%) & $39(31)$ & $63(42)$ & $102(37)$ & 0.0588 \\
\hline Education <12 y, n (\%) & $46(37)$ & $106(71)$ & $152(55)$ & $<0.0001$ \\
\hline \multicolumn{5}{|l|}{ Cardiovascular risk factors } \\
\hline Hypertension, n (\%) & $89(71)$ & $89(60)$ & $178(65)$ & 0.0475 \\
\hline Diabetes mellitus, $\mathbf{n}(\%)$ & $25(20)$ & $21(14)$ & $46(17)$ & 0.19 \\
\hline Dyslipidemia, n (\%) & $54(43)$ & $72(48)$ & $126(46)$ & 0.40 \\
\hline Current smoker, n (\%) & $23(18)$ & $32(21)$ & $55(20)$ & 0.53 \\
\hline Atrial fibrillation, $\mathrm{n}(\%)$ & $24(19)$ & $37(25)$ & $61(22)$ & 0.26 \\
\hline Prior stroke, n (\%) & $16(13)$ & $14(9)$ & $30(11)$ & 0.37 \\
\hline $\mathrm{BMI}, \mathrm{kg} / \mathrm{m}^{2}$ & $26.4 \pm 3.5$ & $27.4 \pm 4.3$ & $27.0 \pm 4.0$ & 0.0265 \\
\hline $\mathrm{SBP}, \mathrm{mm} \mathrm{Hg}$ & $140(132-151)$ & $132(123-147)$ & $136(126-149)$ & 0.0011 \\
\hline $\mathrm{DBP}, \mathrm{mm} \mathrm{Hg}$ & $79(74-84)$ & $82(74-88)$ & $80(74-86)$ & 0.12 \\
\hline \multicolumn{5}{|l|}{ Biochemical measurements } \\
\hline Fasting glucose, $\mathrm{mg} / \mathrm{dL}$ & $100(91-118)$ & $105(94-125)$ & $102(92-121)$ & 0.0960 \\
\hline LDL-C, mg/dL & $132(109-156)$ & $121(100-144)$ & $126(102-149)$ & 0.0095 \\
\hline HDL-C, mg/dL & $48(40-61)$ & $46(39-55)$ & $48(39-57)$ & 0.0912 \\
\hline Triglycerides, mg/dL & $114(90-175)$ & $120(94-166)$ & $117(92-172)$ & 0.71 \\
\hline Serum CRP, mg/dL & $0.4(0.2-0.7)$ & $0.3(0.1-0.6)$ & $0.3(0.1-0.7)$ & 0.0453 \\
\hline Stroke classification, n (\%) & & & & 0.16 \\
\hline Ischemic stroke & $119(95)$ & $143(96)$ & $262(96)$ & \\
\hline Hemorrhagic stroke & $6(5)$ & $6(4)$ & $12(4)$ & \\
\hline Affected territory, $\mathrm{n}(\%)$ & & & & 0.0906 \\
\hline Anterior circulation, left & $35(28)$ & $57(38)$ & $92(34)$ & \\
\hline Anterior circulation, right & $39(31)$ & $49(33)$ & $88(32)$ & \\
\hline Posterior circulation & $45(36)$ & $34(23)$ & $79(29)$ & \\
\hline$>1$ Territory & $6(5)$ & $9(6)$ & $15(5)$ & \\
\hline Etiologic TOAST subtype, $n(\%)$ & & & & 0.46 \\
\hline Large artery atherosclerosis & $21(18)$ & $18(13)$ & $39(15)$ & \\
\hline Cardioembolism & $30(25)$ & $34(24)$ & $64(24)$ & \\
\hline Small artery occlusion & $16(13)$ & $15(10)$ & $31(12)$ & \\
\hline Other etiology & $3(3)$ & $8(6)$ & $11(4)$ & \\
\hline Competing etiology/undefined & $49(41)$ & $68(48)$ & $117(45)$ & \\
\hline \multicolumn{5}{|l|}{ Clinical examination at admission } \\
\hline NIHSS score & $2(1-4)$ & $1(0-2)$ & $2(0-3)$ & $<0.0001$ \\
\hline \multicolumn{5}{|l|}{ Cognitive assessment } \\
\hline IQCODE score & $48.8 \pm 1.9$ & $48.8 \pm 2.4$ & $48.8 \pm 2.2$ & 0.98 \\
\hline
\end{tabular}


Table 1 Baseline characteristics of the 2 study cohorts (continued)

\begin{tabular}{|c|c|c|c|c|}
\hline & DEDEMAS $(n=125)$ & STROKDEM $(n=149)$ & Pooled data $(n=274)$ & $p$ Value \\
\hline MoCA score $<26, \mathrm{n}(\%)$ & $54(43)$ & $65(44)$ & $119(44)$ & 0.94 \\
\hline Time at MoCA administration, $\mathrm{d}$ after admission & $3.0 \pm 1.6$ & $3.1 \pm 0.6$ & $3.1 \pm 1.2$ & 0.48 \\
\hline
\end{tabular}

Abbreviations: $\mathrm{BMI}=$ body mass index; $\mathrm{CRP}=\mathrm{C}$-reactive protein; DBP = diastolic blood pressure; DEDEMAS = Determinants of Dementia After Stroke; HDL-C = high-density lipoprotein cholesterol; IQCODE = Informant Questionnaire on Cognitive Decline in the Elderly; LDL-C = low-density lipoprotein cholesterol; MoCA = Montreal Cognitive Assessment; mRS = modified Rankin Scale; NIHSS = NIH Stroke Scale; SBP = systolic blood pressure; STROKDEM = Study of Factors Influencing Post-Stroke Dementia; TOAST = Trial of Org 10172 in Acute Stroke Treatment.

Values are expressed as number (percent), mean \pm SD, or median (interquartile range).

\section{Results}

\section{Baseline characteristics}

A total of 293 patients with stroke underwent the baseline assessments. Of these 293 patients, 274 (94\%) had an available baseline MoCA score and were included in the analysis. Their baseline characteristics did not differ from those of the overall sample (data available from Dryad, table e-2, doi.org/ $10.5061 /$ dryad.7h083rd). Reasons for noncompletion of the MoCA included exhaustion and aphasia. The most common reasons for missing items were motor deficits and visual impairment (data available from dryad, table e-3, doi.org/10. 5061/dryad.7h083rd).

Table 1 presents the baseline characteristics of the 2 study cohorts $(n=274)$. Compared to patients from STROKDEM, DEDEMAS patients were older and more likely to have hypertension; had higher systolic blood pressure values, lowdensity lipoprotein cholesterol, and C-reactive protein levels but lower body mass index values; and reported a higher educational level. They further had lower NIHSS scores at admission, while prestroke cognitive performance as assessed by the IQCODE did not differ between the 2 cohorts. The majority of index events were classified as ischemic stroke (95\% in DEDEMAS and 96\% in STROKDEM). None of the DEDEMAS patients scored above the threshold for diagnosis of delirium (Delirium Rating Scale-Revised-98 score $\geq 16$ ). Nine patients $(7 \%)$ reported subthreshold symptoms of delirium $(0<$ score $<16)$. MoCA was administered within a mean of $3.0 \pm 1.6$ (range 0-6, DEDEMAS) and $3.1 \pm 0.6$ (range 0-5, STROKDEM) days from stroke onset. The proportion of patients with a MoCA score $<26$ was $43 \%$ in DEDEMAS and 44\% in STROKDEM. Patients with a MoCA score $<26$ were older, less educated, more likely to have hypertension, less likely to be current smokers, more likely to have stroke in the left anterior circulation or in multiple territories, and less likely to have stroke in posterior circulations; had higher baseline glucose and C-reactive protein levels; and had a higher NIHSS score at admission (table 2).

Figure 1 displays the study profile. The proportion of patients who died during the 36-month follow-up period was 9 of 125 (7\%, DEDEMAS) and 12 of 149 (8\%, STROKDEM). Loss to follow-up occurred in 12\% (DEDEMAS) and $17 \%$ (STROKDEM). The total number of assessments for neuropsychological testing, CDR, mRS, and IADL was 615, 678,729 , and 685 , respectively. The baseline characteristics of patients who were lost to follow-up or died did not differ from those in patients who were followed up to 36 months (data available from Dryad, table e-4, doi.org/10.5061/ dryad.7h083rd).

\section{Association of baseline MoCA with study outcomes across the 3-year follow-up interval}

\section{Cognitive and functional outcomes}

A baseline MoCA score $<26$ was associated with cognitive impairment across the 36-month follow-up period (table 3 ). In the fully adjusted pooled analysis, a MoCA score $<26$ was associated with 5.3-fold increased odds of cognitive impairment, defined by a $z$ score $\leq 1.5$ for any cognitive domain in neuropsychological testing (OR 5.30, 95\% confidence interval [CI] 2.75-10.22), and 2.5-fold increased odds of cognitive impairment as defined by a CDR score $\geq 0.5$ (OR 2.53, 95\% CI 1.53-4.18). A MoCA score <26 was further associated with worse cognitive performance in all 4 cognitive domains across the 36-month follow-up period (fully adjusted pooled analysis, table 3 ). Focusing on functional outcome, a baseline MoCA score $<26$ was associated with increased odds of functional impairment, defined by either an $\mathrm{mRS}$ score $>2$ (OR 5.03, 95\% CI 2.20-11.51) or an IADL score $<8$ (OR $2.48,95 \%$ CI $1.40-4.38$ ) in the fully adjusted pooled analysis (table 3).

Similar results were obtained in individual cohorts, except for visuospatial ability in DEDEMAS (effect estimate -0.23 , 95\% CI -0.49 to 0.03 ) and for IADL-defined functional impairment in STROKDEM (OR 1.98, 95\% CI 0.83-4.75, table 3). There was no indication of heterogeneity in the pooled analyses, with the exception of memory (data available from dryad, figure e-1, doi.org/10.5061/dryad.7h083rd). The results remained unchanged when controlled only for age, sex, and education (minimally adjusted models); when analyses were restricted to patients with first-ever stroke; when analyses were restricted to patients with ischemic stroke; when patients with subthreshold delirium symptoms were excluded in DEDEMAS; and when APOE genotype was adjusted for in DEDEMAS (data available from Dryad, table e-5, doi.org/10. 5061/dryad.7h083rd). Similar results were further obtained when individual time points $(6,12$, and 36 months $)$ were 
Table 2 Baseline differences of patients scoring $\leq 26$ and $>26$ on the MoCA within the first week after stroke

\begin{tabular}{|c|c|c|c|}
\hline & MoCA score $\geq 26(n=155)$ & MoCA score $<26(n=119)$ & $p$ Value \\
\hline \multicolumn{4}{|l|}{ Demographic variables } \\
\hline Age, y & $63.9 \pm 11.6$ & $71.1 \pm 9.4$ & $<0.0001$ \\
\hline Female, n (\%) & $53(34)$ & $49(41)$ & 0.24 \\
\hline Education <12 y, n (\%) & $75(48)$ & $77(65)$ & 0.0071 \\
\hline \multicolumn{4}{|l|}{ Cardiovascular risk factors } \\
\hline Hypertension, n (\%) & $93(60)$ & $85(71)$ & 0.0494 \\
\hline Diabetes mellitus, $\mathbf{n}(\%)$ & $22(14)$ & $24(20)$ & 0.19 \\
\hline Dyslipidemia, n (\%) & $65(42)$ & $61(51)$ & 0.12 \\
\hline Current smoker, n (\%) & $39(25)$ & $16(13)$ & 0.0164 \\
\hline Atrial fibrillation, n (\%) & $30(19)$ & $31(26)$ & 0.19 \\
\hline Prior stroke, n (\%) & $17(11)$ & $13(11)$ & 0.99 \\
\hline $\mathrm{BMI}, \mathrm{kg} / \mathrm{m}^{2}$ & $26.9 \pm 4.1$ & $27.1 \pm 3.7$ & 0.64 \\
\hline $\mathrm{SBP}, \mathrm{mm} \mathrm{Hg}$ & $136[124-148]$ & 137 [128-151] & 0.19 \\
\hline DBP, mm Hg & $81[76-87]$ & 79 [72-85] & 0.09 \\
\hline \multicolumn{4}{|l|}{ Biochemical measurements } \\
\hline Fasting glucose, $\mathrm{mg} / \mathrm{dL}$ & $100[91-116]$ & 106 [95-129] & 0.0074 \\
\hline LDL-C, mg/dL & 125 [103-150] & 128 [99-147] & 0.60 \\
\hline HDL-C, mg/dL & 48 [40-57] & $47[39-56]$ & 0.62 \\
\hline Triglycerides, mg/dL & 114 [92-170] & 121 [92-177] & 0.73 \\
\hline Serum CRP, mg/dL & $0.3[0.1-0.6]$ & $0.4[0.2-0.7]$ & 0.0224 \\
\hline Stroke classification, n (\%) & & & 0.07 \\
\hline Ischemic stroke & $152(98)$ & $110(92)$ & \\
\hline Hemorrhagic stroke & $3(2)$ & $9(8)$ & \\
\hline Affected territory, $\mathrm{n}(\%)$ & & & 0.0209 \\
\hline Anterior circulation, left & $48(31)$ & $44(37)$ & \\
\hline Anterior circulation, right & $50(32)$ & $38(32)$ & \\
\hline Posterior circulation & $53(34)$ & $26(22)$ & \\
\hline$>1$ Territory & $4(3)$ & $11(9)$ & \\
\hline Etiologic TOAST subtype, n (\%) & & & 0.16 \\
\hline Large artery atherosclerosis & $19(13)$ & $20(18)$ & \\
\hline Cardioembolism & $35(23)$ & $29(26)$ & \\
\hline Small artery occlusion & $22(14)$ & $9(8)$ & \\
\hline Other etiology & $4(3)$ & $7(6)$ & \\
\hline Competing etiology/undefined & $72(47)$ & $45(41)$ & \\
\hline \multicolumn{4}{|l|}{ Clinical examination at admission } \\
\hline NIHSS score & $1(0-3)$ & $2(1-5)$ & 0.0010 \\
\hline
\end{tabular}


Table 2 Baseline differences of patients scoring $\leq 26$ and $>26$ on the MoCA within the first week after stroke (continued)

\begin{tabular}{llll}
\hline \multicolumn{1}{l}{ MoCA score $\geq \mathbf{2 6}(\mathbf{n}=\mathbf{1 5 5})$} & MoCA score $<\mathbf{2 6}(\mathbf{n}=\mathbf{1 1 9}) \quad$ & \\
\hline Cognitive assessment & & & \\
\hline IQCODE & $48.5 \pm 1.8$ & $49.1 \pm 2.6$ & 0.0549 \\
\hline Time at MoCA administration, d after admission & $3.0 \pm 1.9$ & $2.9 \pm 1.2$ & 0.32 \\
\hline
\end{tabular}

Abbreviations: $\mathrm{BMI}=$ body mass index; $\mathrm{CRP}=\mathrm{C}$-reactive protein; $\mathrm{DBP}=$ diastolic blood pressure; HDL-C = high-density lipoprotein cholesterol; IQCODE = Informant Questionnaire on Cognitive Decline in the Elderly; LDL-C = low-density lipoprotein cholesterol; MoCA = Montreal Cognitive Assessment; $\mathrm{mRS}=$ modified Rankin Scale; NIHSS = NIH Stroke Scale; SBP = systolic blood pressure; TOAST = Trial of Org 10172 in Acute Stroke Treatment.

Values are expressed as number (percent), mean \pm SD, or median (interquartile range).

analyzed (data available from Dryad, table e-6, doi.org/10. 5061/dryad.7h083rd). Because of the overrepresentation of patients with minor stroke in our cohorts (median admission NIHSS score $2[0-3]$ ), we further performed stratified analyses by stroke severity (NIHSS score $\leq 3[\mathrm{n}=199]$ vs $>3[\mathrm{n}=$ 75]) to examine whether these results also apply to patients with more severe stroke. The point estimates for the 2 groups were similar for all outcomes, but the CIs for patients with an NIHSS score $>3$ were wider, possibly because of reduced power (data available from Dryad, figure e-2, doi.org/10. 5061/dryad.7h083rd).

Figure 2 depicts the development of cognitive and functional impairment across the entire follow-up period stratified for

Figure 1 Flowchart of study participants in the DEDEMAS and STROKDEM cohorts

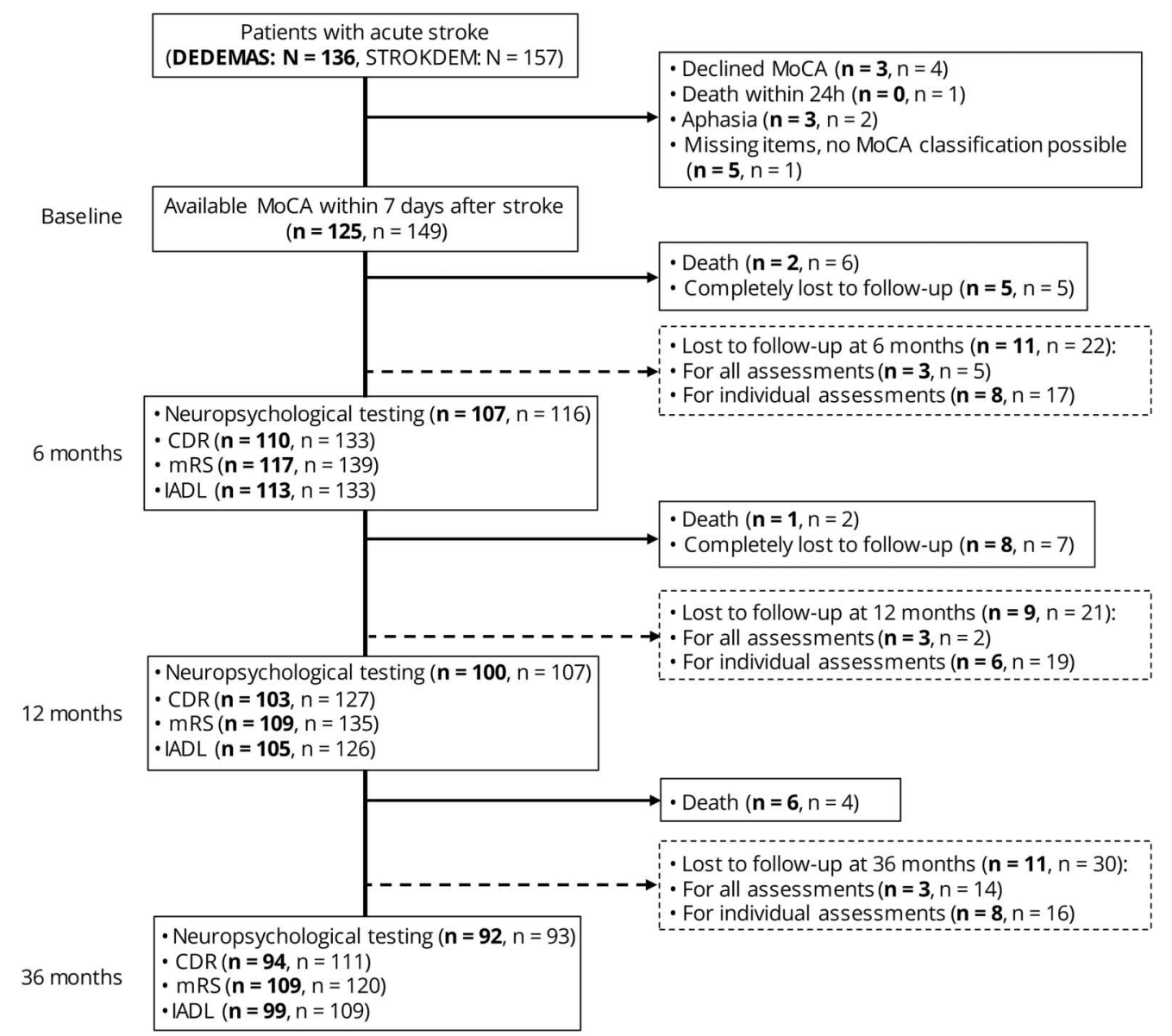

Numbers refer to patients included in Determinants of Dementia After Stroke (DEDEMAS) (bold) and Study of Factors Influencing Post-Stroke Dementia (STROKDEM). Patients were classified as completely lost to follow-up if they declined all further assessments. Patients were defined as lost to follow-up for all assessments if all 4 outcome measures were missing at the corresponding time point. Patients were defined as lost to follow-up for individual assessments if $\geq 1$ assessments were missing at the corresponding time point. CDR = Clinical Dementia Rating; IADL = Instrumental Activities of Daily Living; MoCA = Montreal Cognitive Assessment; mRS = modified Rankin Scale. 
Table 3 Association of baseline MoCA score $(<26 \mathrm{vs} \geq 26)$ within a week after stroke with cognitive and functional outcome and all-cause mortality during up to 3 years of follow-up

\begin{tabular}{|c|c|c|c|c|c|c|}
\hline \multirow[b]{2}{*}{ Cognitive impairment } & \multicolumn{2}{|l|}{ DEDEMAS } & \multicolumn{2}{|l|}{ STROKDEM } & \multicolumn{2}{|l|}{ Pooled data ${ }^{a}$} \\
\hline & OR $(95 \% \mathrm{Cl})$ & $p$ Value & OR $(95 \% \mathrm{Cl})$ & $p$ Value & OR $(95 \% \mathrm{Cl})$ & $p$ Value \\
\hline $\begin{array}{l}\text { Impairment in } \geq 1 \\
\text { domain } \\
\text { (z score } \leq 1.5)\end{array}$ & $5.12(2.01-13.07)$ & 0.0006 & $5.48(2.18-13.80)$ & 0.0003 & $5.30(2.75-10.22)$ & $<0.0001$ \\
\hline CDR score $\geq 0.5$ & $3.22(1.43-7.26)$ & 0.0048 & $2.17(1.14-4.12)$ & 0.0184 & $2.53(1.53-4.18)$ & 0.0003 \\
\hline $\begin{array}{l}\text { Cognitive performance } \\
\text { (mean z score) }\end{array}$ & $\begin{array}{l}\text { Effect estimate } \\
(95 \% \mathrm{Cl})\end{array}$ & $p$ Value & $\begin{array}{l}\text { Effect estimate } \\
(95 \% \mathrm{Cl})\end{array}$ & $p$ Value & $\begin{array}{l}\text { Effect estimate } \\
(95 \% \mathrm{Cl})\end{array}$ & $p$ Value \\
\hline $\begin{array}{l}\text { Executive function/ } \\
\text { attention }\end{array}$ & $-0.74(-1.06$ to -0.42$)$ & $<0.0001$ & $-0.59(-0.88$ to -0.30$)$ & $<0.0001$ & $-0.66(-0.87$ to -0.44$)$ & $<0.0001$ \\
\hline Memory & $-0.34(-0.57$ to -0.12$)$ & 0.0031 & $-0.80(-1.18$ to -0.41$)$ & $<0.0001$ & $-0.54(-0.99 \text { to }-0.09)^{b}$ & 0.0173 \\
\hline Language & $-0.41(-0.65$ to -0.16$)$ & 0.0010 & $-0.42(-0.71$ to -0.13$)$ & 0.0048 & $-0.41(-0.60$ to -0.23$)$ & $<0.0001$ \\
\hline Visuospatial ability & $-0.23(-0.49$ to 0.03$)$ & 0.0855 & $-0.35(-0.61$ to -0.09$)$ & 0.0076 & $-0.29(-0.47$ to -0.11$)$ & 0.0020 \\
\hline Functional impairment & OR $(95 \% \mathrm{Cl})$ & $p$ Value & OR $(95 \% \mathrm{Cl})$ & $p$ Value & OR $(95 \% \mathrm{CI})$ & $p$ Value \\
\hline mRS score $>2$ & $4.75(1.67-13.54)$ & 0.0035 & $5.54(1.44-21.39)$ & 0.0130 & $5.03(2.20-11.51)$ & 0.0001 \\
\hline IADL score $<8$ & $2.93(1.38-6.23)$ & 0.0049 & $1.98(0.83-4.75)$ & 0.1250 & $2.48(1.40-4.38)$ & 0.0018 \\
\hline \multirow[t]{2}{*}{ All-cause mortality } & HR $(95 \% \mathrm{Cl})$ & $p$ Value & $\mathrm{HR}(95 \% \mathrm{Cl})$ & $p$ Value & $\mathrm{HR}(95 \% \mathrm{Cl})$ & $p$ Value \\
\hline & $6.83(0.80-58.53)$ & 0.0796 & $7.48(1.48-37.71)$ & 0.0148 & $7.24(1.99-26.35)$ & 0.0027 \\
\hline
\end{tabular}

Abbreviations: $C D R=$ Clinical Dementia Rating; $C I=$ confidence interval; DEDEMAS = Determinants of Dementia After Stroke; HR = hazard ratio; $I A D L=$ Instrumental Activities of Daily Living; $\mathrm{mRS}$ = modified Rankin Scale; OR = odds ratio; STROKDEM = Study of Factors Influencing Post-Stroke Dementia.

Analyses were adjusted for age, sex, education, history of hypertension, history of diabetes mellitus, NIH Stroke Scale score at admission, and IQCODE score at baseline.

a Effect estimates derived from meta-analysis.

${ }^{\mathrm{b}}$ A random-effects model was used because of heterogeneity $\left(I^{2}=75 \%, p\right.$ value from Cochran $\mathrm{Q}$ test $\left.=0.05\right)$.

patients with a baseline MoCA score $<26$ or $\geq 26$. Patients with a baseline MoCA score $\geq 26$ remained stable between time points (all $p>0.05)$. In contrast, patients with a baseline MoCA score $<26$ showed an increase in the rate of functional impairment defined by an $\mathrm{mRS}$ score $>2$ and an IADL score $<8$ from 12 to 36 months after stroke.

\section{Mortality}

Figure 3 shows the Kaplan-Meier survival curves by baseline MoCA score $(<26$ vs $\geq 26)$. In the Cox regression analysis, a baseline MoCA score <26 was independently associated with increased mortality in the pooled fully adjusted analysis (hazard ratio 7.24, 95\% CI 1.99-26.35, table 2). Similar results were obtained in individual cohorts (table 3 ).

\section{Predictive ability of baseline MoCA for study outcomes across the 3-year follow-up interval}

To determine the additive predictive value of the baseline MoCA on top of age, sex, education, history of hypertension, history of diabetes, IQCODE score at baseline, and NIHSS score at admission, we first calculated the AUCs using the dichotomized MoCA score ( $<26$ vs $\geq 26)$. We found an increase in AUC for cognitive impairment, defined by neuropsychological testing $(0.81$ [95\% CI $0.76-0.85]$ vs 0.72 [95\%
CI 0.67-0.77], $p=0.01)$, and for functional impairment, defined by $\mathrm{mRS}$ score $>2(0.88$ [95\% CI $0.85-0.92$ ] vs 0.84 [95\% CI 0.80-0.87], $p=0.047$, figure 4) but not for CDR score $\geq 0.5$ and IADL score $<8$ (data available from Dryad, table e-7, doi.org/10.5061/dryad.7h083rd). The AUC for mortality was 0.86 (95\% CI 0.77-0.95) in the model including the dichotomized MoCA score ( $<26$ vs $\geq 26)$ and 0.80 (95\% CI $0.70-0.89)$ in a model not including it ( $p=0.09$, figure 4$)$.

We next repeated the analyses with the entire range of baseline MoCA scores as a continuous variable $(n=262)$. Compared with the dichotomized MoCA scores, there was no further improvement of the AUC for any of the outcomes. Furthermore, the ROC curves for the continuous MoCA confirmed 25 of 26 as the optimal cutoff point for the prediction of cognitive impairment, defined by neuropsychological testing, and for functional impairment, defined by an $\mathrm{mRS}$ score $>2$ (data available from Dryad, table e-8, doi.org/10.5061/dryad.7h083rd).

\section{Discussion}

This study shows that cognitive screening by the MoCA administered within the first week after stroke onset adds to the prediction of cognitive outcome, functional outcome, and 
A

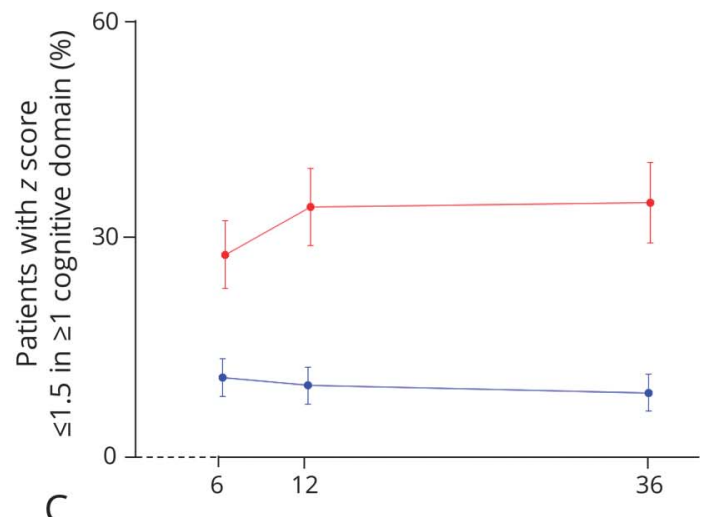

C

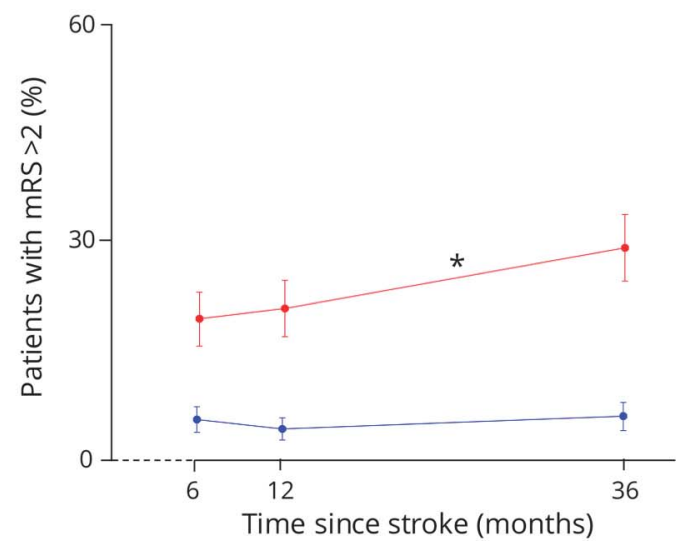

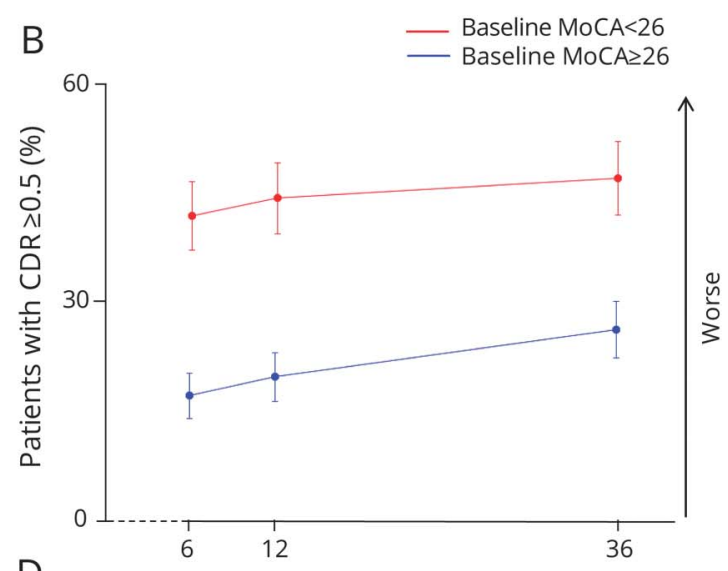

D

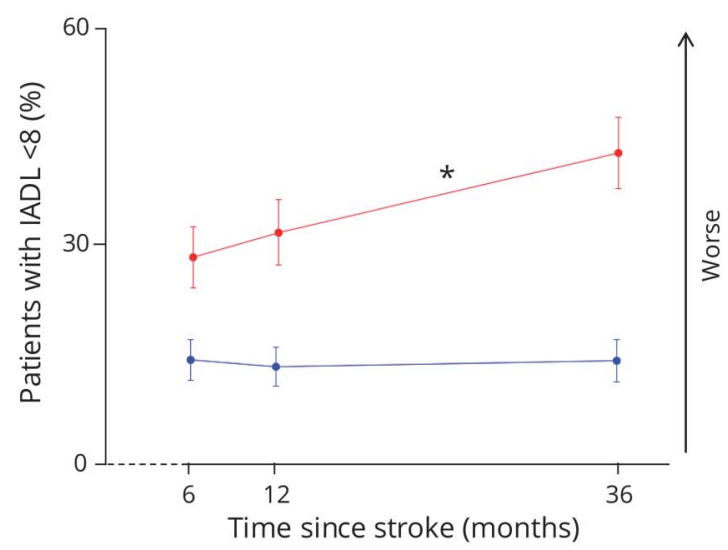

Percentages of patients with (A) impairment in at least 1 cognitive domain ( $z$ score $\leq 1.5)$, (B) Clinical Dementia Rating (CDR) score $\geq 0.5$, (C) modified Rankin Scale (mRS) score $>2$, and (D) impairment in Instrumental Activities of Daily Living (IADL) score $<8$ during the follow-up assessments of the pooled Determinants of Dementia After Stroke (DEDEMAS) and Study of Factors Influencing Post-Stroke Dementia (STROKDEM) sample. Error bars correspond to standard errors. MoCA = Montreal Cognitive Assessment. *Statistically significant changes (false discovery rate $<0.05$ ) between time points.

mortality up to 3 years after the event. Specifically, we found that patients without prestroke dementia scoring $<26$ on the MoCA at baseline were at increased risk for cognitive impairment defined by comprehensive neuropsychological assessment and CDR, functional impairment assessed by the $\mathrm{mRS}$ and IADL, and all-cause mortality. Across the 3-year follow-up interval, patients with a MoCA score <26 performed worse in multiple cognitive domains, including executive function/attention, memory, language, and visuospatial ability. The results were remarkably consistent across 2 independent cohorts and were stable in sensitivity analyses. Collectively, our findings emphasize the clinical utility of early cognitive testing after stroke.

The baseline MoCA added to outcome prediction independently of other prognostic factors, including age, premorbid cognitive status, and, most notably, stroke severity as assessed by the NIHSS score at admission. The NIHSS is an established predictor of functional outcome after stroke $e^{37,38}$ and has been associated with early mortality after stoke. ${ }^{39}$ However, it lacks a cognitive component, ${ }^{40}$ and its relationship with cognitive outcomes is disputed. ${ }^{41,42}$ In the current study, inclusion of the admission NIHSS score without baseline MoCA did not add to the prediction of cognitive impairment and mortality. In contrast, adding the baseline MoCA score to the admission NIHSS score led to an increase in the $C$ statistic for all outcomes, including functional outcome, across the 3 years of follow-up, thus demonstrating the clinical utility of cognitive screening after stroke.

The use of a dichotomized MoCA at the previously validated threshold of $<26^{10,24,25}$ allowed us to classify patients with missing items due to focal neurologic deficits. Previous studies testing patients in the first days after stroke found the MoCA to be infeasible in $\approx 20 \%$ of participants possibly because they made no attempts to classify patients with missing items. $^{12-18,43}$ In contrast, we found $94 \%$ of our patients to be classifiable. We further found the dichotomized score to perform as well as the continuous score in predicting longterm outcomes. Moreover, ROC curve analyses identified the threshold of $<26$ as optimal for predicting cognitive and functional impairment. Overall, these results support the use of the threshold of $<26$ for cognitive testing after stroke. Still, $6 \%$ of our patients could not be classified or declined investigation. While not explored in detail, these patients showed a high mortality ( 7 of 19 patients [37\%] after 3 years), 
Figure 3 Kaplan-Meier survival curves by baseline MoCA score

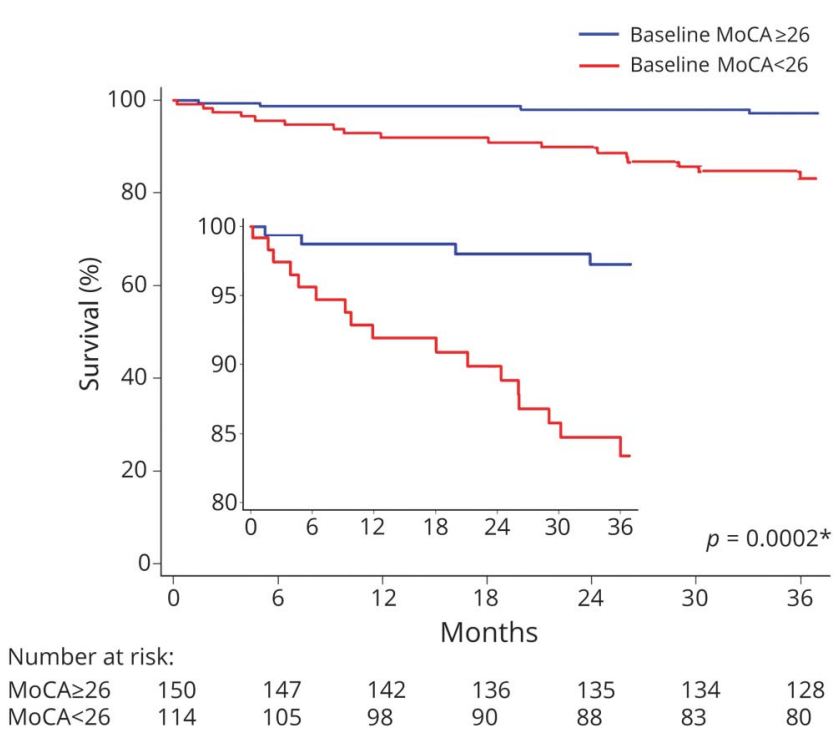

Shown are the results from the pooled Determinants of Dementia After Stroke (DEDEMAS) and Study of Factors Influencing Post-Stroke Dementia (STROKDEM) sample. MoCA = Montreal Cognitive Assessment. *Log-rank test comparing the 2 survival curves.

which would be in agreement with our findings in testable patients and with earlier studies. ${ }^{15,43}$

Aside from being predictive of poor cognitive outcome, a baseline MoCA score $<26$ was associated with worse functional outcome as illustrated by an increase in the proportion of patients with functional impairment between 12 and 36 months after stroke. This may in part relate to the known influence of cognitive deficits on measures of disability and performance in daily activities. ${ }^{6,44}$ Alternatively, the decline in functional performance might relate to limitations in health care delivery. Patients with poststroke cognitive impairment have been shown to be at higher risk for poor adherence to treatment guidelines and to have restricted access to rehabilitation programs despite evidence for considerable functional gains in this patient group. ${ }^{44}$ Hence, a MoCA score $<26$ might identify patients requiring special attention.

We also found the MoCA at baseline to predict 3-year mortality. This fits with previous studies that found cognitive deficits 3 months after stroke ${ }^{7}$ and incident poststroke dementia ${ }^{45}$ to be associated with higher mortality. Extending these observations, our study shows that cognitive testing in the very first days after stroke adds to the prediction of mortality, thus enabling the identification of high-risk patients. As the Kaplan-Meier curves demonstrated, the mortality rates were relatively constant across the 3-year follow-up period both in patients with a MoCA score $<26$ and in those with a MoCA score $\geq 26$. Future studies exploring the causes of death may help us understand the reasons for poor prognosis in patients with a baseline MoCA score $<26$.
The rates of cognitive impairment both in the total sample (defined by neuropsychological testing) and in those with a baseline MoCA score <26 remained rather stable between 12 and 36 months. While this suggests a stabilization of cognitive performance 1 year after stroke on a group level in patients with mostly mild stroke and no prestroke dementia, this finding needs to be interpreted cautiously. Some patients might have deteriorated while others improved because of ongoing disease or repair processes, respectively. In addition, we might have lost patients who deteriorated during follow-up.

Our results support the feasibility and clinical utility of the MoCA within the first week after stroke despite practical challenges. ${ }^{20,21}$ The MoCA has retained validity even the first days after stroke, ${ }^{12,13}$ takes $<10$ minutes to complete, ${ }^{46}$ and is feasible in the majority of patients with stroke. ${ }^{12,13}$ It has high sensitivity for mild cognitive impairment in stroke patients, ${ }^{11}$ is especially sensitive to executive deficits that are common in vascular cognitive impairment, ${ }^{10}$ and better reflects underlying vascular pathology than the widely used Mini-Mental State Examination. ${ }^{25,47}$ Hence, there are strong arguments for routine testing of stroke patients with the MoCA early after hospital admission.

This study has several methodologic strengths. Our results were derived from 2 independent cohorts of stroke patients recruited in different countries. Harmonization of study protocols between DEDEMAS and STROKDEM enabled pooled analyses, which enhanced power. Our study had a long follow-up of 3 years and included a wide range of outcomes comprehensively assessed by multiple methods. Serial measurements at 6,12 , and 36 months enabled longitudinal analyses with generalized estimating equations models providing precise estimates. Finally, we accounted for known confounders and predictors of poor stroke outcome either by study design or in multivariable analysis, thus determining the predictive ability of the baseline MoCA on top of other factors.

Our study also has limitations. First, our study might not be fully representative of stroke in general because both cohorts excluded patients with prestroke dementia and recruited predominantly patients with mild stroke, who were more likely to consent to study inclusion. This resulted in a high proportion of cases with low NIHSS scores, which limited the variance of the NIHSS and might hence underestimate its predictive value in the models and overestimate the predictive value of MoCA, especially for predicting functional impairment. The selection of mildly affected patients is also reflected by the high survival rate 3 years after stroke (91\%), which is closer to the rates reported for minor stroke ${ }^{48}$ than for stroke in general. ${ }^{49}$ Although our results were similar even among the small subsample of patients with admission NIHSS score $>3$, they should be cautiously interpreted and require confirmation by future studies with a larger representation of patients with major stroke. On the other hand, 

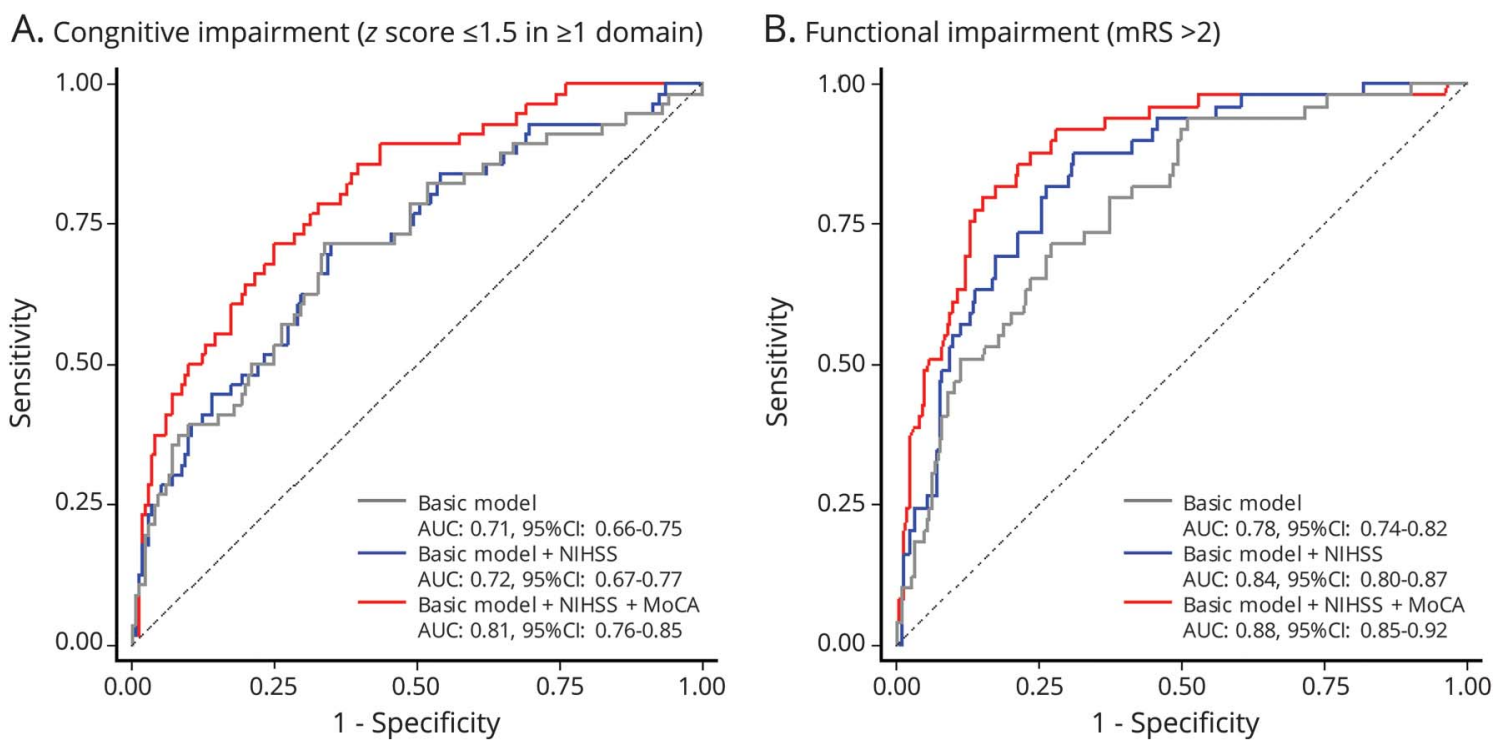

C. All-cause mortality

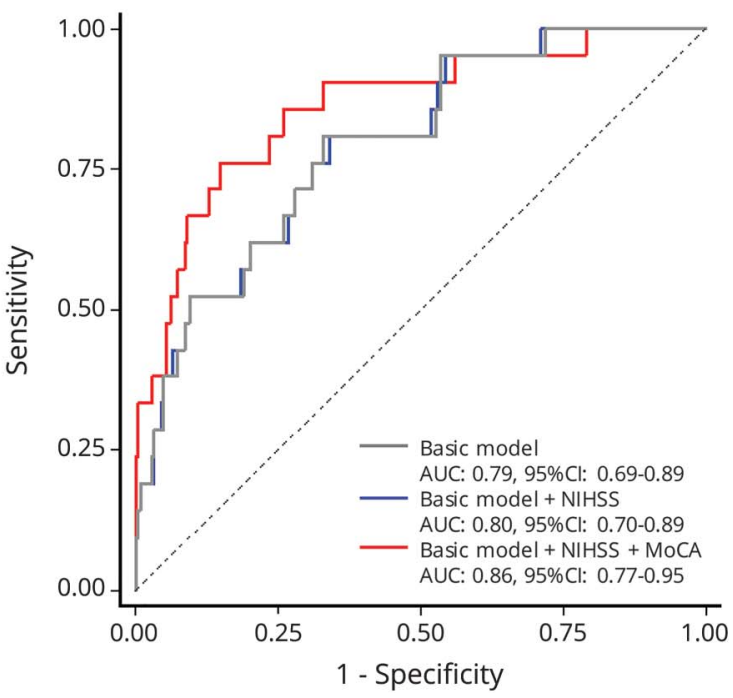

Receiver operating characteristic (ROC) curves were derived from 3 additive models predicting (A) cognitive impairment as defined by neuropsychological testing, (B) functional impairment as defined by the modified Rankin Scale (mRS) scores, and (C) all-cause mortality during the 3-year follow-up period. The basic model was adjusted for age, sex, education, history of hypertension, history of diabetes mellitus, and Informant Questionnaire on Cognitive Decline in the Elderly at baseline; the additional models further include NIH Stroke Scale (NIHSS) score at admission (continuous scale) and Montreal Cognitive Assessment (MoCA) at admission (dichotomized; score $<26 \mathrm{vs} \geq 26$ ). Shown are the results from the pooled Determinants of Dementia After Stroke (DEDEMAS) and Study of Factors Influencing Post-Stroke Dementia (STROKDEM) sample. AUC = area under the ROC curve.

our sample is representative of patients who are most likely to benefit from targeted prevention. Second, we cannot exclude a bias resulting from attrition. Patients not examined by extensive face-to-face visits during follow-up are more likely to have dementia, ${ }^{50}$ and this could lead to lower rates of cognitive impairment when assessed by detailed neuropsychological testing at follow-up. Third, the MoCA was still not feasible in a small proportion of patients at baseline $(8 \%$ in DEDEMAS and $5 \%$ in STROKDEM) despite dichotomization. Fourth, we could not control for delirium in STROKDEM. However, we expect the rate of delirium in STROKDEM to be very low because none of the patients in DEDEMAS met the criteria for delirium. Finally, prestroke cognitive function was assessed by the informant-based IQCODE questionnaire rather than formal testing. Therefore, residual confounding of prestroke cognitive function remains possible.

This study shows that the MoCA administered within the first week after stroke is a strong predictor of long-term cognitive outcome, functional outcome, and mortality with added predictive value on top of established predictors. Given the brevity of the test and its feasibility in the setting of acute stroke, our findings support the use of the MoCA as a routine clinical tool to identify high-risk patients who might benefit from close monitoring. 


\section{Author contributions}

M. Dichgans, V. Zietemann, and M. Georgakis designed the study, developed the protocol, obtained ethics approval for the DEMDAS study, and wrote the first draft of the manuscript. F.A. Wollenweber, C. Müller, and A. Kopczak were involved in patient recruitment, acquisition of data, and revising the manuscript. V. Zietemann and M. Georgakis performed the data analysis, and V. Zietemann, M. Georgakis, and M. Dichgans interpreted the data. R. Bordet, A.-M. Mendyk, and H. Hénon designed the STROKDEM study, developed the protocol, organized logistical aspects, and obtained ethics approval for STROKDEM. T. Dondaine performed the neuropsychological assessment. A.-M. Mendyk monitored the data. H. Hénon, S. Bombois, and R. Bordet were involved in recruitment and adjudication of patients. A.-M. Mendyk and T. Dondaine prepared the database. All authors reviewed and edited the manuscript and approved the final version of the manuscript.

\section{Acknowledgment}

The authors thank Margit Deschner, Brigitte Huber, Julia Hill, Rupert Aigner, Antonia Weingart, and Didier Leys for their support.

\section{Study funding}

This work was supported by the Deutsche Forschungsgemeinschaft (German Research Foundation) within the framework of the Munich Cluster for Systems Neurology (EXC 1010 SyNergy), the German Center for Neurodegenerative Diseases, Munich, the European Union's Horizon 2020 research and innovation program under grant agreement 666881, SVDs@target, and the Vascular Dementia Research Foundation. STROKDEM was supported by the French Health Ministry and Fondation Coeur et Artère.

\section{Disclosure}

The authors report no disclosures relevant to the manuscript. Go to Neurology.org/ $\mathrm{N}$ for full disclosures.

\section{Publication history}

Received by Neurology March 14, 2018. Accepted in final form August 1, 2018.

\section{References}

1. Benjamin EJ, Blaha MJ, Chiuve SE, et al. Heart disease and stroke statistics-2017 update: a report from the American Heart Association. Circulation 2017;135:e146-e603.

2. Feigin VL, Lawes CM, Bennett DA, Anderson CS. Stroke epidemiology: a review of population-based studies of incidence, prevalence, and case-fatality in the late 20th century. Lancet Neurol 2003;2:43-53.

3. Jaillard A, Naegele B, Trabucco-Miguel S, LeBas JF, Hommel M. Hidden dysfunctioning in subacute stroke. Stroke 2009;40:2473-2479.

4. Pendlebury ST, Rothwell PM. Prevalence, incidence, and factors associated with prestroke and post-stroke dementia: a systematic review and meta-analysis. Lancet Neurol 2009;8:1006-1018.

5. Nys GM, van Zandvoort MJ, van der Worp HB, et al. Early cognitive impairment predicts long-term depressive symptoms and quality of life after stroke. J Neurol Sci 2006;247:149-156.

6. Nys GM, van Zandvoort MJ, de Kort PL, et al. The prognostic value of domain specific cognitive abilities in acute first-ever stroke. Neurology 2005;64: $821-827$.

7. Oksala NK, Jokinen H, Melkas S, et al. Cognitive impairment predicts poststroke death in long-term follow-up. J Neurol Neurosurg Psychiatry 2009;80:1230-1235.

8. Quinn TJ, Elliott E, Langhorne P. Cognitive and mood assessment tools for use in stroke. Stroke 2018;49:483-490.
9. Nasreddine ZS, Phillips NA, Bedirian V, et al. The Montreal Cognitive Assessment, MoCA: a brief screening tool for mild cognitive impairment. J Am Geriatr Soc 2005; 53:695-699.

10. Pendlebury ST, Cuthbertson FC, Welch SJ, Mehta Z, Rothwell PM. Underestimation of cognitive impairment by Mini-Mental State Examination versus the Montreal Cognitive Assessment in patients with transient ischemic attack and stroke: a population-based study. Stroke 2010;41:1290-1293.

11. Burton L, Tyson SF. Screening for cognitive impairment after stroke: a systematic review of psychometric properties and clinical utility. J Rehabil Med 2015;47: 193-203.

12. Horstmann S, Rizos T, Rauch G, Arden C, Veltkamp R. Feasibility of the Montreal Cognitive Assessment in acute stroke patients. Eur J Neurol 2014;21:1387-1393.

13. Pasi M, Salvadori E, Poggesi A, Inzitari D, Pantoni L. Factors predicting the Montreal Cognitive Assessment (MoCA) applicability and performances in a stroke unit. J Neurol 2013;260:1518-1526.

14. Dong Y, Venketasubramanian N, Chan BP, et al. Brief screening tests during acute admission in patients with mild stroke are predictive of vascular cognitive impairment 3-6 months after stroke. J Neurol Neurosurg Psychiatry 2012;83: $580-585$.

15. Salvadori E, Pasi M, Poggesi A, Chiti G, Inzitari D, Pantoni L. Predictive value of $\mathrm{MoCA}$ in the acute phase of stroke on the diagnosis of mid-term cognitive impairment. J Neurol 2013;260:2220-2227.

16. Jacquin $\mathrm{A}$, Binquet $\mathrm{C}$, Rouaud $\mathrm{O}$, et al. Post-stroke cognitive impairment: high prevalence and determining factors in a cohort of mild stroke. J Alzheimers Dis 2014; 40:1029-1038.

17. Dong $\mathrm{Y}, \mathrm{Xu}$ J, Chan BP, et al. The Montreal Cognitive Assessment is superior to National Institute of Neurological Disease and Stroke-Canadian Stroke Network 5 -minute protocol in predicting vascular cognitive impairment at 1 year. BMC Neurol 2016;16:46.

18. Dong Y, Slavin MJ, Chan BP, et al. Cognitive screening improves the predictive value of stroke severity scores for functional outcome 3-6 months after mild stroke and transient ischaemic attack: an observational study. BMJ Open 2013;3: e003105

19. Wollenweber FA, Zietemann V, Rominger A, et al. The Determinants of Dementia After Stroke (DEDEMAS) study: protocol and pilot data. Int J Stroke 2014;9: 387-392.

20. Ponchel A, Labreuche J, Bombois S, Delmaire C, Bordet R, Henon H. Influence of medication on fatigue six months after stroke. Stroke Res Treat 2016;2016:2410921.

21. Jorm AF, Korten AE. Assessment of cognitive decline in the elderly by informant interview. Br J Psychiatry 1988;152:209-213.

22. Brott T, Adams HP Jr, Olinger CP, et al. Measurements of acute cerebral infarction: a clinical examination scale. Stroke 1989;20:864-870.

23. Adams HP Jr, Bendixen BH, Kappelle LJ, et al. Classification of subtype of acute ischemic stroke: definitions for use in a multicenter clinical trial: TOAST: Trial of Org 10172 in Acute Stroke Treatment. Stroke 1993;24:35-41.

24. Lees R, Selvarajah J, Fenton C, et al. Test accuracy of cognitive screening tests for diagnosis of dementia and multidomain cognitive impairment in stroke. Stroke 2014; 45:3008-3018.

25. Zamboni G, Griffanti L, Jenkinson M, et al. White matter imaging correlates of early cognitive impairment detected by the Montreal Cognitive Assessment after transient ischemic attack and minor stroke. Stroke 2017;48:1539-1547.

26. Knopman DS, Beiser A, Machulda MM, et al. Spectrum of cognition short of dementia: Framingham Heart Study and Mayo Clinic Study of Aging. Neurology 2015; 85:1712-1721.

27. Hughes CP, Berg L, Danziger WL, Coben LA, Martin RL. A new clinical scale for the staging of dementia. Br J Psychiatry 1982;140:566-572.

28. Woolf C, Slavin MJ, Draper B, et al. Can the Clinical Dementia Rating scale identify mild cognitive impairment and predict cognitive and functional decline? Demen Geriatr Cogn Disord 2016;41:292-302.

29. Rankin J. Cerebral vascular accidents in patients over the age of 60, II: prognosis. Scott Med J 1957;2:200-215.

30. Ganesh A, Luengo-Fernandez R, Wharton RM, et al. Time course of evolution of disability and cause-specific mortality after ischemic stroke: implications for trial design. J Am Heart Assoc 2017;6:e005788.

31. Synhaeve NE, Schaapsmeerders P, Arntz RM, et al. Cognitive performance and poor long-term functional outcome after young stroke. Neurology 2015;85: 776-782.

32. Lawton MP, Brody EM. Assessment of older people: self-maintaining and instrumental activities of daily living. Gerontologist 1969;9:179-186.

33. Mok VC, Wong A, Lam WW, et al. Cognitive impairment and functional outcome after stroke associated with small vessel disease. J Neurol Neurosurg Psychiatry 2004; 75:560-566.

34. Mayeda ER, Tchetgen Tchetgen EJ, Power MC, et al. A simulation platform for quantifying survival bias: an application to research on determinants of cognitive decline. Am J Epidemiol 2016;184:378-387.

35. Fischer U, Baumgartner A, Arnold M, et al. What is a minor stroke? Stroke 2010;41: 661-666.

36. Higgins JPT, Green S. Cochrane Handbook for Systematic Reviews of Interventions Version 5.1.0 (updated March 2011) [online]. Available at: cochrane-handbook.org. Accessed June 28, 2015.

37. Adams HP Jr, Davis PH, Leira EC, et al. Baseline NIH Stroke Scale score strongly predicts outcome after stroke: a report of the Trial of Org 10172 in Acute Stroke Treatment (TOAST). Neurology 1999;53:126-131. 
38. Lees KR, Bluhmki E, von Kummer R, et al. Time to treatment with intravenous alteplase and outcome in stroke: an updated pooled analysis of ECASS, ATLANTIS, NINDS, and EPITHET trials. Lancet 2010;375:1695-1703.

39. Nam HS, Kim HC, Kim YD, et al. Long-term mortality in patients with stroke of undetermined etiology. Stroke 2012;43:2948-2956.

40. Kasner SE. Clinical interpretation and use of stroke scales. Lancet Neurol 2006;5: 603-612.

41. Srikanth VK, Anderson JF, Donnan GA, et al. Progressive dementia after first-ever stroke: a community-based follow-up study. Neurology 2004;63:785-792.

42. Altieri M, Di Piero V, Pasquini M, et al. Delayed poststroke dementia: a 4-year followup study. Neurology 2004;62:2193-2197.

43. Pendlebury ST, Klaus SP, Thomson RJ, et al. Methodological factors in determining risk of dementia after transient ischemic attack and stroke: (III) applicability of cognitive tests. Stroke 2015;46:3067-3073.

44. Rabadi MH, Rabadi FM, Edelstein L, Peterson M. Cognitively impaired stroke patients do benefit from admission to an acute rehabilitation unit. Arch Phys Med Rehabil 2008;89:441-448.
45. Desmond DW, Moroney JT, Sano M, Stern Y. Mortality in patients with dementia after ischemic stroke. Neurology 2002;59:537-543.

46. Quinn TJ, Elliott E, Langhorne P. Cognitive and mood assessment tools for use in stroke. Stroke 2018;49:483-490.

47. Pasi M, Salvadori E, Poggesi A, et al. White matter microstructural damage in small vessel disease is associated with Montreal Cognitive Assessment but not with Mini Mental State Examination performances: Vascular Mild Cognitive Impairment Tuscany study. Stroke 2015;46:262-264.

48. Prencipe M, Culasso F, Rasura M, et al. Long-term prognosis after a minor stroke: 10 year mortality and major stroke recurrence rates in a hospital-based cohort. Stroke 1998;29:126-132.

49. Wolfe CD, Crichton SL, Heuschmann PU, et al. Estimates of outcomes up to ten years after stroke: analysis from the prospective South London Stroke Register. PLoS Med 2011;8:e1001033.

50. Pendlebury ST, Chen PJ, Welch SJ, et al. Methodological factors in determining risk of dementia after transient ischemic attack and stroke: (II) effect of attrition on followup. Stroke 2015;46:1494-1500. 


\section{Neurology}

Early MoCA predicts long-term cognitive and functional outcome and mortality after stroke

Vera Zietemann, Marios K. Georgakis, Thibaut Dondaine, et al.

Neurology 2018;91;e1838-e1850 Published Online before print October 17, 2018

DOI 10.1212/WNL.0000000000006506

This information is current as of October 17, 2018

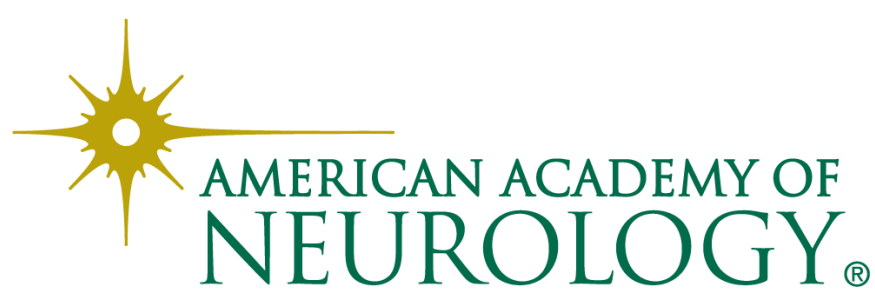




\section{Updated Information \& Services}

References

Citations

Subspecialty Collections

\section{Permissions \& Licensing}

\section{Reprints}

including high resolution figures, can be found at: http://n.neurology.org/content/91/20/e1838.full

This article cites 49 articles, 28 of which you can access for free at: http://n.neurology.org/content/91/20/e1838.full\#ref-list-1

This article has been cited by 2 HighWire-hosted articles: http://n.neurology.org/content/91/20/e1838.full\#\#otherarticles

This article, along with others on similar topics, appears in the following collection(s):

\section{All Cerebrovascular disease/Stroke}

http://n.neurology.org/cgi/collection/all_cerebrovascular_disease_strok e

All Rehabilitation

http://n.neurology.org/cgi/collection/all_rehabilitation

Assessment of cognitive disorders/dementia

http://n.neurology.org/cgi/collection/assessment_of_cognitive_disorder S_dementia

Natural history studies (prognosis)

http://n.neurology.org/cgi/collection/natural_history_studies_prognosis

Neuropsychological assessment

http://n.neurology.org/cgi/collection/neuropsychological_assessment

Information about reproducing this article in parts (figures,tables) or in its entirety can be found online at:

http://www.neurology.org/about/about_the_journal\#permissions

Information about ordering reprints can be found online:

http://n.neurology.org/subscribers/advertise

Neurology ${ }^{\circledR}$ is the official journal of the American Academy of Neurology. Published continuously since 1951, it is now a weekly with 48 issues per year. Copyright (O 2018 American Academy of Neurology. All rights reserved. Print ISSN: 0028-3878. Online ISSN: 1526-632X.

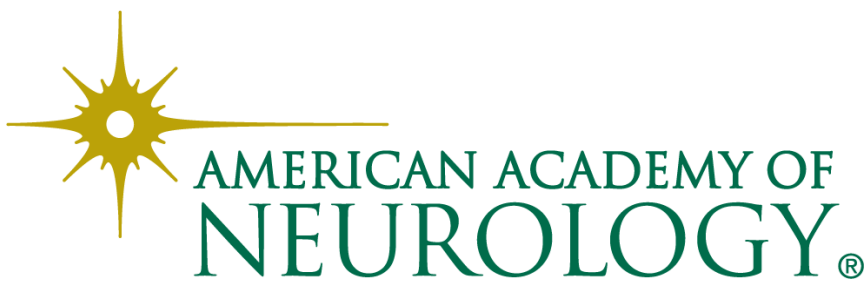

